\title{
Article
}

\section{Reaching One Single and Stable Critical Cluster through Finite-Sized Systems}

\author{
Romain Grossier, and Ste\#phane Veesler
}

Cryst. Growth Des., Article ASAP • Publication Date (Web): 27 February 2009

Downloaded from http://pubs.acs.org on February 27, 2009

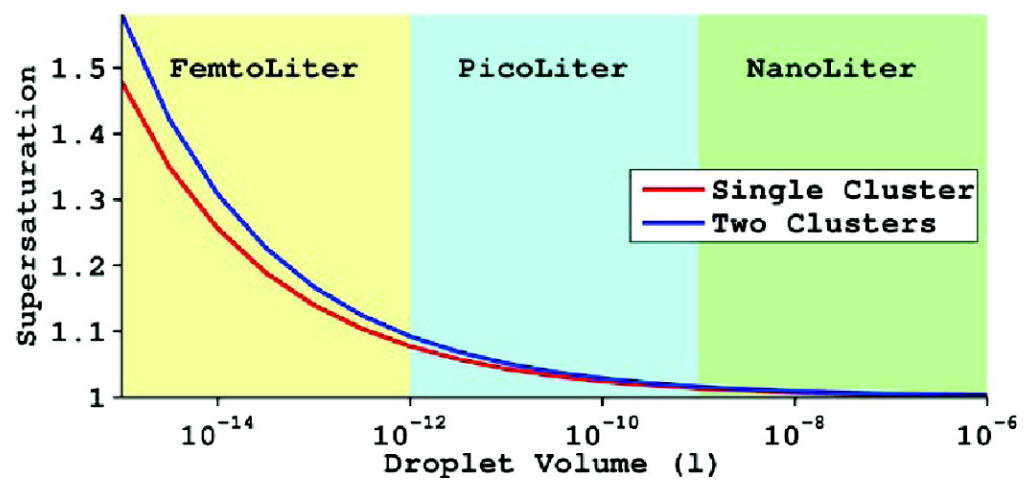

\section{More About This Article}

Additional resources and features associated with this article are available within the HTML version:

- $\quad$ Supporting Information

- $\quad$ Access to high resolution figures

- $\quad$ Links to articles and content related to this article

- Copyright permission to reproduce figures and/or text from this article

View the Full Text HTML 


\title{
Reaching One Single and Stable Critical Cluster through Finite-Sized Systems
}

\author{
Romain Grossier* and Stéphane Veesler* \\ Centre Interdisciplinaire de Nanoscience de Marseille (CINaM), CNRS, Campus de Luminy, \\ Case 913, 13288 Marseille Cedex 09, France
}

Received October 17, 2008; Revised Manuscript Received December 21, 2008

\begin{abstract}
Despite a century of active research on first-order phase transitions, discrepancies between predictions based on nucleation theory and experiments on nucleation rates are still of several orders of magnitude. This is partly due to the way the work needed to create a critical cluster is modeled. Here, using slightly modified classical nucleation theory, we reconsider confinement effect leading to one single and stable critical cluster. We relate the new cluster equilibrium size arising from confinement to the usual critical cluster size in infinite systems. The single and stable critical cluster opens new experimental horizons: it can be studied in detail. We stress the model-free nature of these results.
\end{abstract}

\section{Introduction}

Although first-order phase transitions can produce beauty in everyday life (snowflakes, ${ }^{1}$ dew, clouds, champagne bubbles), their extensive study is certainly driven by their vital role in numerous industrial processes (synthesis of inorganic nanoparticles, ${ }^{2}$ pharmaceutics ${ }^{3}$ ), in vivo processes, ${ }^{4}$ or meteorology ${ }^{5}$ predictions. A century of active research has led to the emergence of a generic two-step mechanism: a cluster of the new phase first has to nucleate in the older phase before growing. The underlying reason why a system undergoes transition is the energy gain this allows: the system is in a metastable state and tries to reach a more favorable equilibrium state. The energy released by each molecule passing from the old phase to the new phase will be proportional to the chemical potential difference (the driving force of transition) between the old and new phases: $\Delta \mu=\mu_{\text {old }}-\mu_{\text {new,eq, }}$, with $\mu_{\text {new,eq }}$ the chemical potential of the new phase at equilibrium. However, for the birth of the new phase, work has to be done, at a minimum to create an interface between the two phases. This nucleation work acts as a barrier to transition, so that the cluster of the new phase has first to reach the critical cluster size $n^{*}$. Below this critical size, the cluster is more likely to dissolve; above this critical size it is more likely to grow. This partially explains the stochasticity of nucleation, and the inherent induction time before the emergence of the new phase.

Excess Energy: A Tricky Problem. In a system undergoing first-order phase transition under constant external pressure $P$ and constant temperature $T$, the characteristic thermodynamic potential is the Gibbs free energy $G$. The change of Gibbs free energy ${ }^{6}$ associated with the formation of a cluster of size $n$ (the number of molecules) can then be expressed in the general form

$$
\Delta G(n)=-n \Delta \mu+G_{\text {excess }}(n)
$$

The first term of eq 1 is the Gibbs free energy gain if the cluster was just a part of the (new) bulk phase. The second term is an excess term taking into account deviations of the properties of the cluster from bulk phase: the interface with the old phase, homogeneity or heterogeneity of the nucleation process, or differences in the structure; all model assumptions can be encompassed by this excess term. Expressing $G_{\text {excess }}$ is one of

* Corresponding author. E-mail: grossier@ cinam.univ-mrs.fr (R.G.); veesler@ cinam.univ-mrs.fr (S.V.). the trickiest problems in nucleation theories, and unfortunately vital: the probability that a spontaneous fluctuation will give rise to the new phase directly depends on the work $\Delta G^{*}=$ $\Delta G\left(n^{*}\right)$, needed to form a cluster of critical size. In trying to overcome this problem, different thermodynamic formulations of eq 1 have been developed, ${ }^{7}$ all having their own limitations. The most obvious problem is how to accurately express the dependence of interfacial energy relative to the curvature ${ }^{8}$ imposed by the extremely tiny size of precritical and critical clusters. Another limitation arises particularly in crystallization: structure $^{9-12}$ undoubtedly has great impact on the nucleation work.

Difficulties in modeling first-order phase transitions do not only come from the problems encountered to accurately express $G_{\text {excess }}$. This is particularly valuable for nucleation of crystals: pre-exponential terms in kinetic formulations are difficult to express to fit collected data, because of the fact that one needs two-order parameters to properly describe transitions in such systems. Specially designed nucleation theory have then been elaborate..$^{13}$ The objective of here presented study is to show how to bring experiments at the scale of a single critical cluster, with the simple aid of finite sized systems to collect data on $G_{\text {excess }}$. Thereby, we prefer using simple expressions for $G_{\text {excess }}$ and classical nucleation theory, a combination that has the advantage to be easily understandable.

Thus, to test the accuracy of nucleation models, there is a pressing need for experiments at the critical cluster scale. In the following, we present how confined (i.e., finite-sized) systems can furnish a single and stable critical cluster, ideal for detailed study. We must now choose an "experimentalaccessible" first-order phase transition at the scale of its critical cluster. We choose the crystallization of proteins in solution because they combine both a substantial individual size (ranging from several nanometers to several hundred nanometers) and long time scales, making them more accessible to experimental technologies than other classes of matter (atomic or molecular systems). Because of the huge quantity of available data, ${ }^{14}$ we chose lysozyme. The solution, a small droplet, is the following: water, $\mathrm{pH} 4.5, \mathrm{NaCl}(4 \% \mathrm{w} / \mathrm{v}), \mathrm{NaAc}(0.1 \mathrm{M})$ (for lysozyme solubility see).${ }^{15}$ Results are calculated for the temperature $T$ $=293.15 \mathrm{~K}$, so that solubility is equal to $3.1 \mathrm{mg} / \mathrm{mL}$. The interfacial energy $\gamma$ is here taken to be $\gamma=1 \mathrm{~mJ} \mathrm{~m}^{-2} \cdot{ }^{16}$ As we express the size of a cluster via the number $n$ of proteins that 
constitute it, we also need the volume of an individual lysozyme protein, which is $v_{0}=29.7 \mathrm{~nm}^{3} .{ }^{17}$

A Simple Confinement Model. For an approach that is both generic and simple, applicable to all first-order phase transitions, we choose the classical nucleation theory. In a solution at supersaturation $\beta_{0}$, the differential form of Gibbs free energy (eq 1) due to an infinitesimal change in size of an " $n$-sized cluster" in an infinite system can be expressed as follows

$$
d G_{\infty}(n)=-k_{\mathrm{B}} T \ln \left(\beta_{0}\right) d n+\frac{2}{3} A n^{-1 / 3} d n
$$

with $k_{\mathrm{B}}$ the Boltzman constant and $n$ the size, in number of proteins, of the associated cluster. The supposed spherical shape of the " $n$-sized cluster" and the interfacial energy are taken into account through use of $A=4 \pi \gamma\left(\left(3 v_{0}\right) /(4 \pi)\right)^{2 / 3}$. A hypothesis of the classical nucleation theory is the infinite sized system: the supersaturation is considered as a constant all along the emergence of an " $n$-sized cluster". Under this set of hypothesis, integration of eq 2 gives the total decrease in Gibbs free energy for an " $n$-sized cluster"

$$
\Delta G_{\infty}(n)=-n k_{\mathrm{B}} T \ln \left(\beta_{0}\right)+A n^{2 / 3}
$$

From eq 3, an analytical expression of the classical critical cluster size $n^{*}$ in an infinite system, here labeled $n^{*}$, can be obtained

$$
n^{*}\left(\beta_{0}\right)=\frac{8 A^{3}}{27\left(k_{\mathrm{B}} T \ln \left(\beta_{0}\right)\right)^{3}}
$$

In a confined system (a small droplet), supersaturation $\beta_{0}$ can no longer be taken as a constant, and must be corrected, throughout the evolution of an " $n$-sized cluster": once a molecule join the new emerging cluster, it no longer contributes to the supersaturation of the solution. In other words, supersaturation decreases irrespective of the evolution of the " $n$-sized cluster", and the real supersaturation as experienced by this cluster is expressed as

$$
\beta(n)=\left(\beta_{0} N_{\mathrm{s}}-n\right) / N_{\mathrm{s}}
$$

The size of the system appears via the $N_{\mathrm{s}}$ term, the number of proteins a droplet has at saturation, the equilibrium state. We then reformulate eq 2 with the aid of eq 5

$$
d G_{\mathrm{conf}}(n)=-k_{\mathrm{B}} \ln \left(\frac{\beta_{0} N_{\mathrm{s}}-n}{N_{\mathrm{s}}}\right) d n+\frac{2}{3} A n^{-1 / 3} d n
$$

The total decrease in Gibbs free energy due to the emergence of an " $n$-sized cluster" in a system of size $N_{\mathrm{s}}$ is then given by

$$
\Delta G_{\text {conf }}(n)=-k_{\mathrm{B}} T \int_{0}^{n} \ln \left(\frac{\beta_{0} N_{\mathrm{s}}-n}{N_{\mathrm{s}}}\right) d n+A n^{2 / 3}
$$

Due to the use of the evolving supersaturation $\beta(n)$, an analytical form of the critical cluster associated to confined systems, can no longer be determine. Equivalence between $\Delta G_{\text {conf }}(n)$ (eq 7) and $\Delta G_{\infty}(n)$ (eq 3 ) is found when the size of the system tends to infinite values $\left(N_{\mathrm{s}} \rightarrow \infty\right)$. In other words, the critical cluster size found via $\Delta G_{\text {conf }}(n)$ (eq 7) and $\Delta G_{\infty}(n)$ (eq 3) corresponds for high $N_{\mathrm{s}}$ values.

For all the figures presented in this paper, we choose to represent results from $\Delta G_{\text {conf }}(n)$ (eq 7) with solid symbols, and

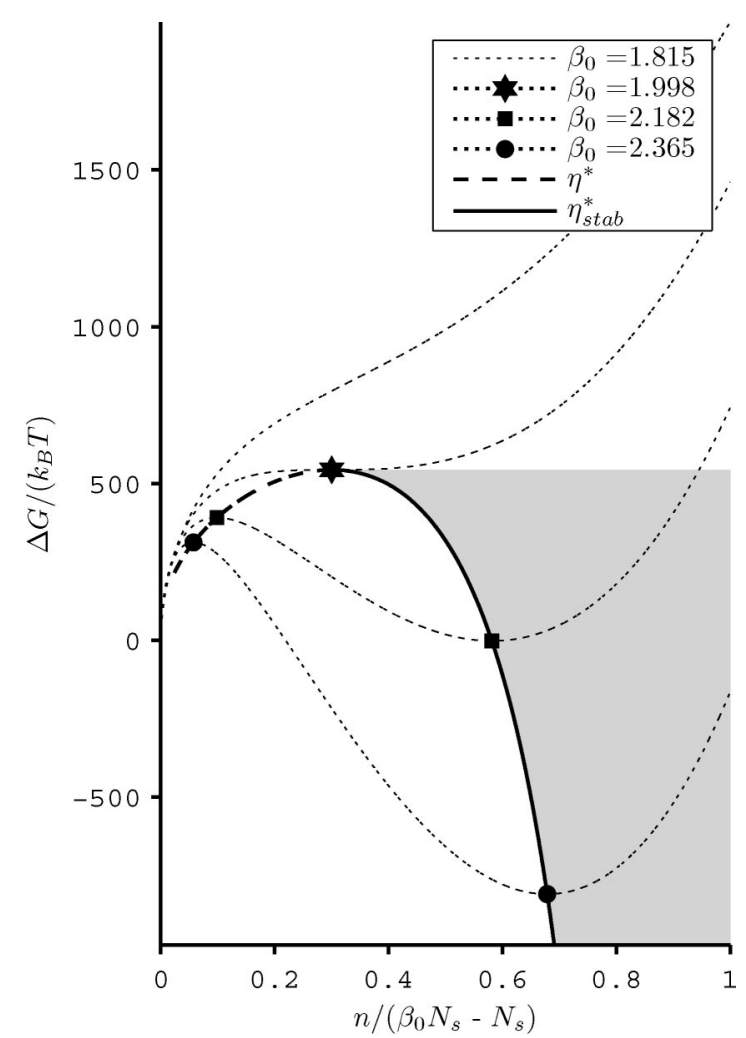

Figure 1. Gibbs free energy ( $\Delta G_{\text {conf }}$ ) evolution during formation of an " $n$-sized cluster" for different initial supersaturations $\beta_{0}$.

open symbols for results from $\Delta G_{\infty}(n)$ (eq 3). Moreover, any given symbol represents the same point in each figure.

This homemade expression of $\Delta G_{\text {conf }}(n)$ (equation 7) shows confinement effects directly. If we choose to express the size of the system by using $N_{\mathrm{s}}$, this is to get a clearer view than the volume parameter allows: the evolution of $\Delta \mu$ during the nucleation and growth process will be directly related to the "weight" $\left(1 / N_{\mathrm{s}}\right)$ a single protein has on it. If we take the example of a femtoliter droplet, this could be an infinite system for a highly soluble protein (high $N_{\mathrm{s}}$ ), opposed to a weakly soluble protein (low $N_{\mathrm{s}}$ ). This also demonstrate the importance of temperature: for protein for which solubility is sensitive to temperature, the same volume droplet could be an infinite (at a temperature where solubility is high) or finite (at a temperature where solubility is low) system.

Confinement Produces Stable Cluster. Confinement (small volume systems) effects have already demonstrated interesting features both in condensation of droplets in superheated vapor $^{18-21}$ and in crystallization in solution. ${ }^{22,23}$ These authors focus on kinetics or crystal habit under the confinement constraint. Our goal is different: we are interested in the stable cluster due to confinement effects. Figure 1 describes this stable cluster, as already pointed out. ${ }^{18-23}$ We first plot the evolution of $\Delta G_{\text {conf }}(n)$, along the formation of an " $n$-sized cluster", for four different increasing initial supersaturation $\beta_{0}$ in a droplet of size $N_{\mathrm{s}}=1 \times 10^{4}$ (corresponding here to a 0.78 femtoliter droplet). The size $n$, on the abscissa, has been adimensionalized: $\beta_{0} N_{\mathrm{s}}$ being the total number of proteins in the droplet, $\beta_{0} N_{\mathrm{s}}-$ $N_{\mathrm{s}}$ is the number of excess proteins available for phase transition, i.e., the maximum size a crystal should reach. Indeed, $n /\left(\beta_{0} N_{\mathrm{s}}\right.$ $-N_{s}$ ) will vary from 0 (no supersaturation has been consumed) to 1 (all the supersaturation has been consumed).

On the basis of figures similar to Figure 1, previous descriptions of first-order phase transitions in finite systems 
marked out two zones. The first zone is below a critical initial supersaturation called $\beta_{\text {crit }}$, here $\beta_{0}=1.998$, where no nucleation can occur. This is due to the evolution of supersaturation throughout the growth of a precritical cluster: it tries to a reach critical size, but depletion of the solution decreases the supersaturation, thus increasing the critical size that needs to be reach. The precritical cluster chases the critical size, and can never catch it. The second zone is for supersaturation above $\beta_{0}=$ 1.998. Here, the cluster has been sufficiently boosted by a high starting supersaturation, to reach and overtake the "moving" critical size, labeled $\eta^{*}$. Once critical size $\eta^{*}$ has been overtaken, the cluster growth continues to decrease the supersaturation in the solution, leading to the appearance of a potential well before thermodynamical equilibrium is reached: the solution is still supersaturated, but the cluster cannot grow larger a size, labeled $\eta_{\text {stab }}$. Evolution of both $\eta^{*}$ and $\eta_{\text {stab }}$ with increasing values of $\beta_{0}$ are shown in Figure 1. The corresponding residual supersaturation, according to eq $5, \beta\left(\eta_{\mathrm{stab}}\right)$, is represented by the shaded zone in Figure 1.

The Stable Cluster Is Critical Too. In this representation (Figure 1), two important results can be seen. First, there is a third zone inside which, although a potential well $\eta_{\text {stab }}$ actually exists, it does not represent a gain in Gibbs free energy for the system. On Figure 1, it corresponds to $1.998<\beta_{0} \leq 2.182$. The limit value $\beta_{0}=2.182$ is interesting in that the system has equal probabilities of being in one of the two configurations: energy is the same, and $\Delta G_{\text {conf }}\left(\eta_{\text {stab }}\right)=0$. This can provide a direct measurement of the work associated with the creation of a critical cluster of size $\eta^{*}$.

Second, both the labile equilibrium $\eta^{*}$ and the stable cluster $\eta_{\text {stab }}$ are the classical critical cluster in infinite system $n^{*}$, according to equation 3 calculated for the "instantaneous" supersaturation, noted $\beta\left(\eta^{*}\right)$ and $\beta\left(\eta_{\text {stab }}\right)$ respectively, as expressed by eq 5 . This allows a correspondence between finite and infinite systems, as summarized by the following equalities

$$
\begin{gathered}
\eta^{*}=n^{*}\left(\beta\left(\eta^{*}\right)\right) \\
\eta_{\text {stab }}=n^{*}\left(\beta\left(\eta_{\text {stab }}\right)\right) \Rightarrow \eta_{\text {stab }}^{*}
\end{gathered}
$$

These points are the locus where partial derivates, irrespective to $n$, of eqs 7 and 3 (for supersaturation calculated from eq 5) equals zero. Equation 9 shows that the stable cluster $\eta_{\text {stab }}$ is in fact a critical cluster, so that we now label $\eta_{\text {stab }}$ as $\eta_{\text {stab }}^{*}$.

Figure 2 supports this result. A plot of $\Delta G_{\text {conf }}(n)$ for an initial supersaturation $\beta_{0}=2.182$ has been drawn in comparison with three plots of $\Delta G_{\infty}(n)$, for $\beta_{0}=2.182, \beta\left(\eta^{*}\right)$, and $\beta\left(\eta_{\text {stab }}^{*}\right)$. Figure 2 has been adimensionalized compared to the classical case: $n / n^{*}$ in abscissa, and $\Delta G(n) / \Delta G_{\infty}^{*}$ for the ordinates. We then find at $(1,1)$, represented as an open square in Figure 2, the classical critical cluster size $n^{*}$ and its energy barrier height $\Delta G_{\infty}^{*}$, as calculated from eq 3 . Two vertical solid lines clearly show the equivalence in size between extrema of the finite system and labile equilibrium of the infinite one, as stated by eqs 8 and 9. This equivalence holds true for any supersaturation $\beta \geq \beta_{\text {crit. }}$

The Critical Cluster Curve Is Folded. Correspondence between finite and infinite systems is more than just an equivalence in size. Both extrema $\eta^{*}$ and $\eta_{\text {stab }}^{*}$ are of the same essence: they are critical clusters. This may, at first sight, appear astonishing, given the instability of the first and the stability of the second. But this difference is due to the fact that the critical cluster size curve is folded: the finiteness of the system gives rise to an unstable branch $\left(\eta^{*}\right)$ and a stable branch $\left(\eta_{\text {stab }}^{*}\right)$. This statement is explained with the help of Figure 3, which focuses

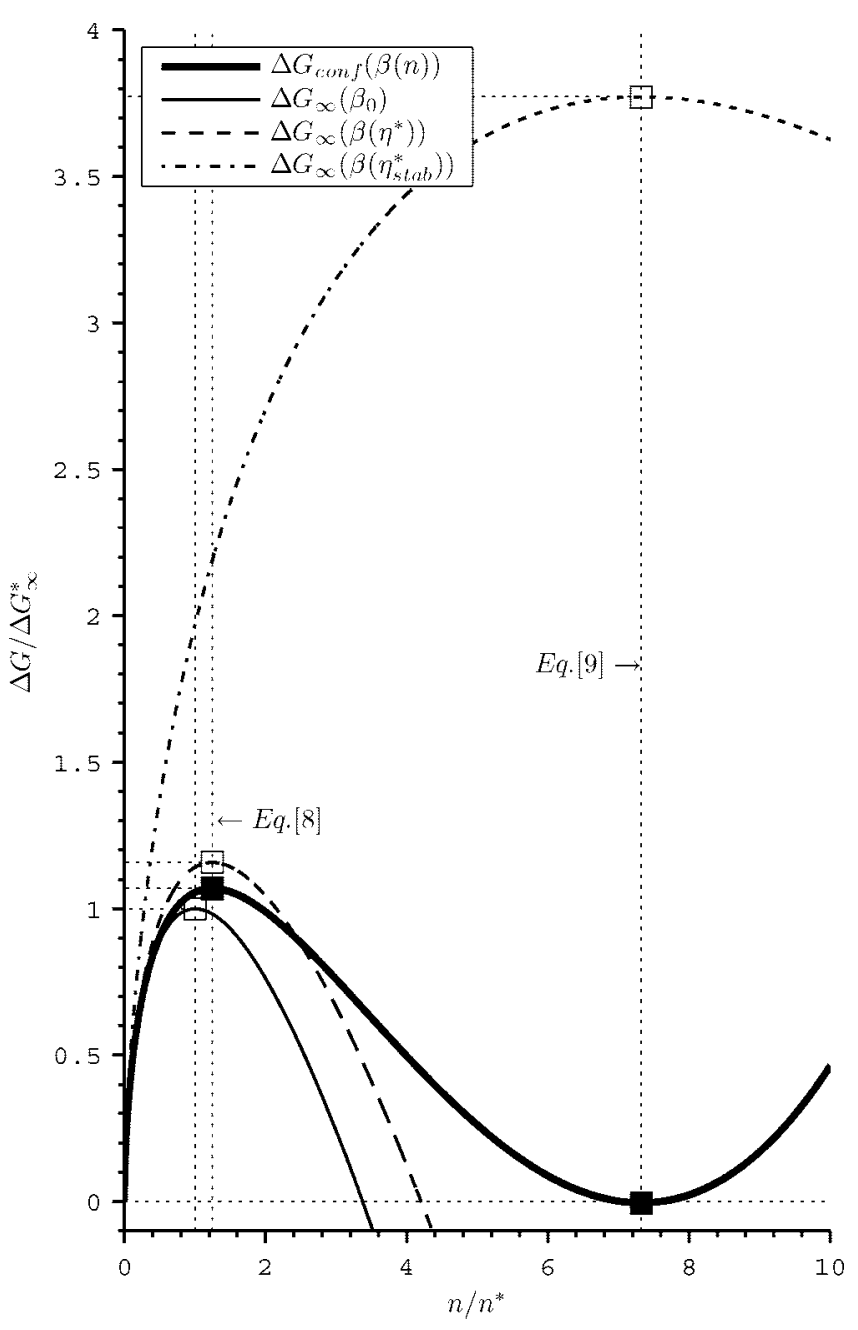

Figure 2. Correspondence of labile $\eta^{*}$ and stable $\eta_{\text {stab }}^{*}$ equilibrium in finite system with infinite systems.

on the area of interest. On this figure, we first plot the classical $n^{*}$-curve of infinite systems. Then we plot sizes of $\eta^{*}$ and $\eta_{\text {stab }}^{*}$ versus initial supersaturation $\beta_{0}$, as calculated and already presented in Figure 1. The curve $\beta_{0} N_{\mathrm{s}}-N_{\mathrm{s}}$ is the number of excess proteins versus $\beta_{0}$. The shaded area, above the $\eta_{\text {stab }}^{*}$ curve corresponds to the shaded area in Figure 1: this is the residual supersaturation $\beta\left(\eta_{\text {stab }}^{*}\right)$. To clearly show that finite system extrema come from the folding of infinite critical cluster size curve $n^{*}$, possible intersections of $\beta(n)$ with the $n^{*}$ curve must be taken into account. We plot $\beta(n)$, namely the supersaturation experienced by the cluster during its evolution, for the same set of initial supersaturations as Figure 1 , for $0 \leq n \leq \beta_{0} N_{\mathrm{s}}-$ $N_{\text {s. }}$.

For $\beta(n)$ with $\beta_{0}=1.814$, which is smaller than $\beta_{\text {crit }}$, we see that it is impossible for the system to generate even a single critical cluster: $\beta(n)$ never intersects the $n^{*}$ curve. For $\beta(n)$ with $\beta_{0}=1.998$, which is exactly $\beta_{\text {crit }}$, the curve $\beta(n)$ intersects and is tangent to the $n^{*}$ curve at one single point. Yet, for the two other $\beta(n)$ curves, plotted for $\beta_{0}=2.182$ and 2.366, the critical cluster can be overtaken: this is the location of the first intersection point with the $n^{*}$ curve, corresponding to $\eta^{*}$. By overtaking, a cluster can grow, thus still decreasing $\beta(n)$, leading to a second intersection point with $n^{*}$, corresponding to $\eta_{\text {stab }}^{*}$ : the growing cluster has caught up with its critical size. Horizontal lines connecting similar open and solid symbols show correspondence between finite and infinite systems as expressed by eqs 8 and 9 . 


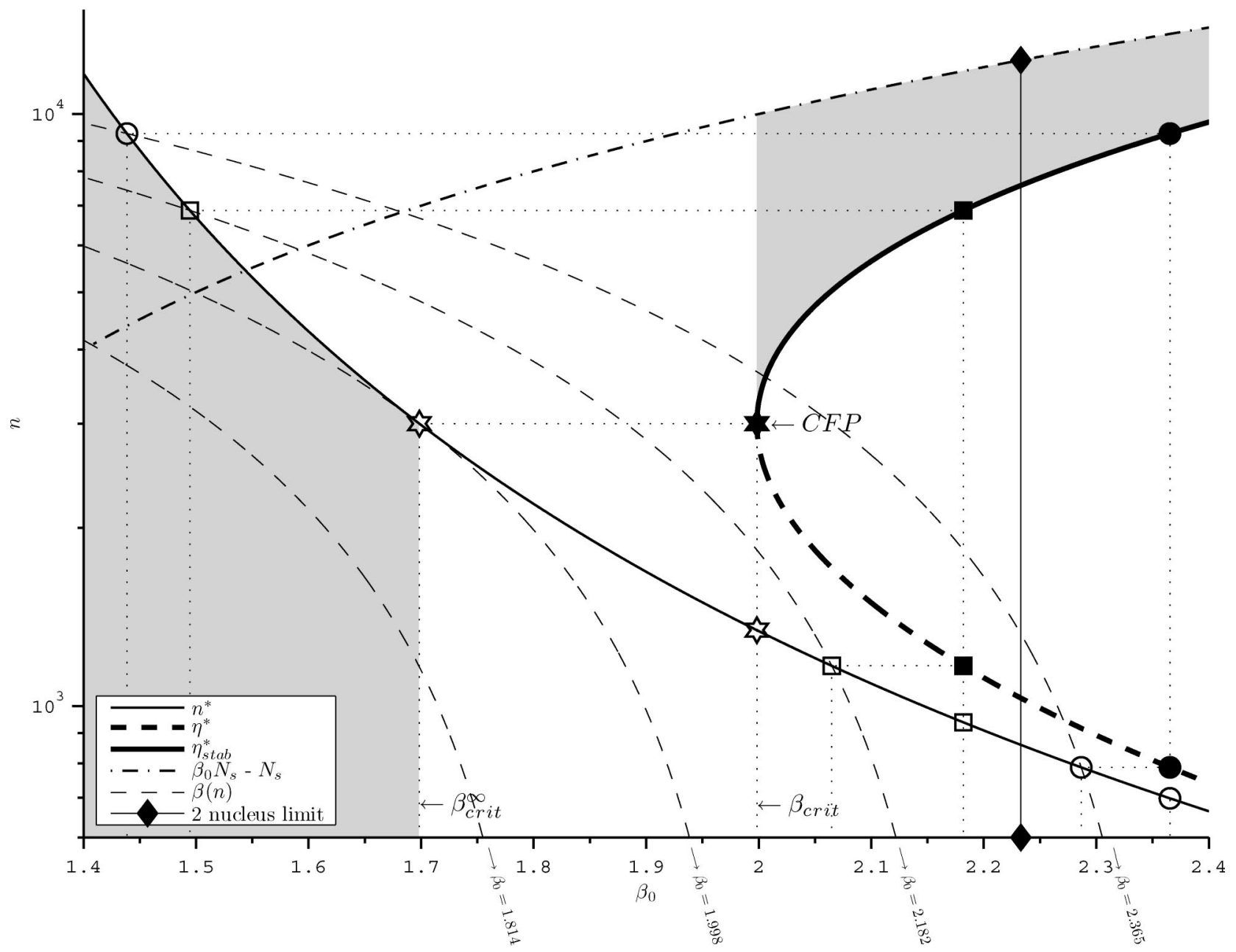

Figure 3. Folded critical cluster size curve for a finite system, and correspondence with an infinite system. Please note that axes do not start at zero, so that vertical dotted lines do not clearly appear to be the starting value, for $n=0$, of $\beta(n)$ lines.

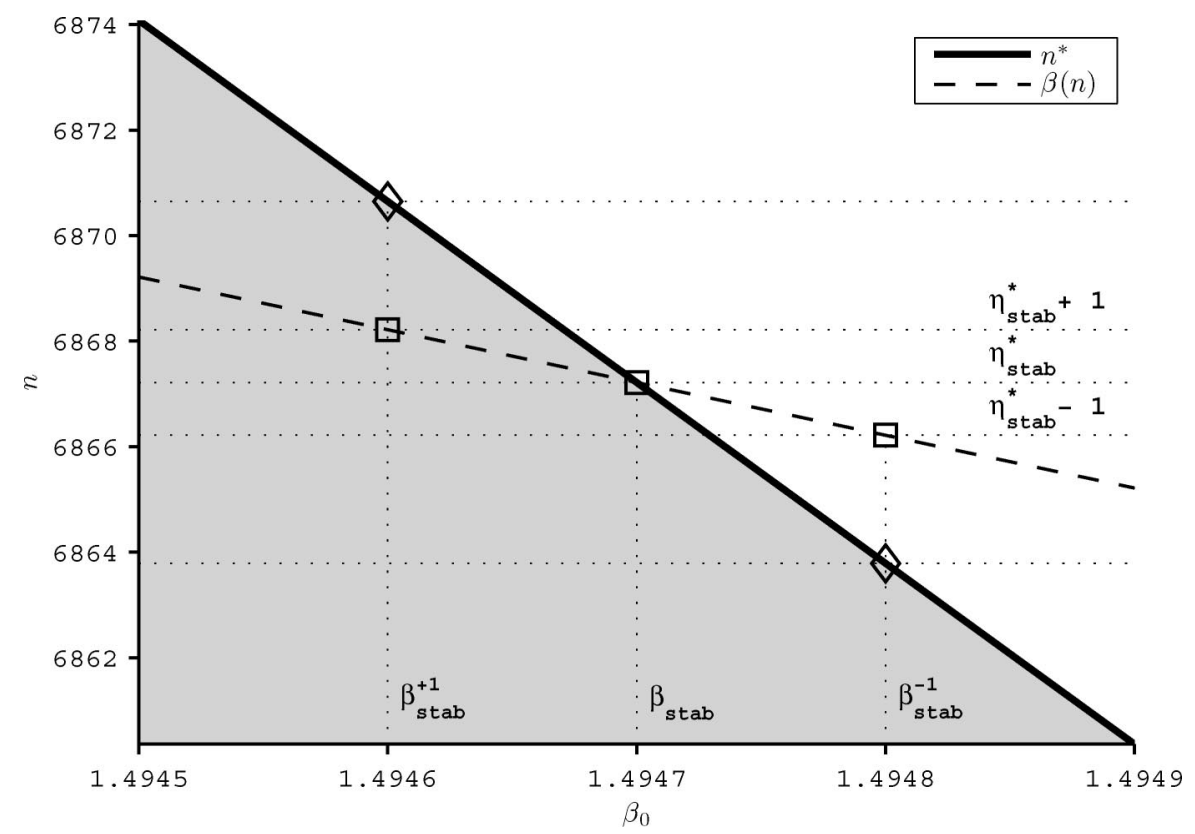

Figure 4. Zoom of Figure 3 on the second intersection point of the $\beta(n)$ curve with the $n^{*}$ curve, calculated for $\beta_{0}=2.182$.

The usual interpretation of the $n^{*}$-curve is as follows: each supersaturation $\beta_{0}$ has a corresponding critical cluster required to overcome, $n^{*}$. But this curve can also be interpreted as an equilibrium curve: to compensate for the finite size of a cluster, the system must always be supersaturated. The smaller a cluster is, the greater the supersaturation must be. This is the driving 
force for Oswald ripening. The importance of this equilibrium interpretation is fully illustrated in its application to finite systems: the system, in nucleating a single cluster, must, once critical size $\eta^{*}$ has been overtaken, always keep sufficient supersaturation to balance the finite size of this cluster. This can not be done for clusters larger than $\eta_{\text {stab. This double }}^{*}$ interpretation of the $n^{*}$ curve leads here to $\eta^{*}$ and $\eta_{\text {stab }}^{*}$ curves, which once joined give the folded $n^{*}$ curve.

The Critical Folding Point. We here define the critical folding point (CFP) as the point where $\eta^{*}=\eta_{\text {stab }}^{*}$, thus corresponding to $\beta_{\text {crit. }}$ CFP location directly depends on the size $N_{\mathrm{s}}$ of the system. Considering points of the classical critical cluster size curve, we determine the corresponding CFP supersaturation in the infinite system using

$$
\frac{\partial n^{*}}{\partial \beta_{0}}=-N_{\mathrm{s}}
$$

This relation can be seen in Figure 3, at the point represented as an open hexagram, where the $n^{*}$ curve is tangent to the $\beta(n)$ curve, whose slope is " $-N_{\mathrm{s}}$ ". Resolution of eq 10 gives the supersaturation $\beta_{\text {crit }}^{\infty}$, the equivalent supersaturation of CFP in the infinite system. Clusters corresponding to higher supersaturations on $n^{*}$ curve will give rise to the unstable branch $\eta^{*}$. Below that critical supersaturation, clusters give rise to the stable branch $\eta_{\text {stab. }}^{*}$. In other words, the $n^{*}$ curve is separated into two parts: one where the first intersection with the $\beta(n)$ curve occurs, and the other where the second intersection occurs. Because eq 10 gives us the supersaturation $\beta_{\text {crit }}^{\infty}$, we thus know the associated critical cluster size $n^{*}$, calculated with eq 4: $n^{*}\left(\beta_{\text {crit }}^{\infty}\right)$. The corresponding initial supersaturation $\beta_{\text {crit }}$ in the " $N$ s-sized system" is found using eq 5 for $n=n^{*}$, so that we determine $\left(\beta_{\text {crit }}, n^{*}\right)$, the location of CFP: the solid hexagram in Figure 3. Equation 10 gives the smallest cluster that can be stabilized in an " $N_{\mathrm{s}}$-sized system", and use of eq 5 directly gives $\beta_{\text {crit, }}$ the initial supersaturation below which nucleation cannot occur.

A Simple Way to Construct the Folded Curve. Up to now, we have plotted the folded critical cluster size curve of the " $N_{\mathrm{s}}$-sized system" from $\Delta G_{\text {conf }}$, according to eq 7. But here we propose a simpler and faster method. This method only relies on $n^{*}$ (equation 4), $\beta(n)$ (equation 5) and knowledge of size correspondence (eqs 8 and 9): each $n^{*}$ has a corresponding single point on the folded curve, either on the stable or the unstable branch. $\beta\left(n^{*}\right)$ can therefore be simply expressed from eq 5 , giving

$$
\beta_{0}=\beta\left(n^{*}\right)+\frac{n^{*}}{N_{\mathrm{s}}}
$$

Thus, for any size $n^{*}$ there is a corresponding initial supersaturation $\beta_{0}$ in the " $N_{\mathrm{s}}$-sized system". The folded critical cluster size curve is the plot of $\left(\beta_{0}, n^{*}\right)$ points, exactly superimposed on the two branches $\eta^{*}$ and $\eta_{\text {stab }}^{*}$ in Figure 3. This clearly shows that both extrema emerging from confinement are of the same essence: they are critical clusters.

To support, once again, the affirmation that the stable cluster $\eta_{\text {stab }}^{*}$ is critical, we provide Figure 4, which is a zoom of Figure 3 on the second intersection point of $\beta(n)$ curve with the $n^{*}$ curve, calculated for $\beta_{0}=2.182$. At this intersection point, cluster $\eta_{\text {stab }}^{*}$ is in equilibrium with a solution at supersaturation $\beta_{\text {stab. }}$. We represent two other points corresponding to the addition and the substraction of a single protein to the cluster of size $\eta_{\text {stab. }}^{*}$ This gives supersaturations $\beta_{\text {stab }}^{+1}$ and $\beta_{\text {stab }}^{-1}$ corresponding to sizes $\eta_{\text {stab }}^{*}+1$ and $\eta_{\text {stab }}^{*}-1$, respectively. To evidence that $\eta_{\text {stab }}^{*}$ is a critical cluster, we propose the following experiment: if we seed the cluster $\eta_{\text {stab }}^{*}$ in an infinite solution at supersaturation $\beta_{\text {stab}}^{+1}$, it is undercritical, so that it dissolves; if we seed the cluster $\eta_{\text {stab }}^{*}$ in an infinite solution at supersaturation $\beta_{\text {stab }}^{-1}$, it is now overcritical, so that it grows; last, if we seed the cluster $\eta_{\text {stab }}^{*}$ in an infinite solution at supersaturation $\beta_{\text {stab }}$, it is critical, so that it can either grow or dissolve. Thus, the cluster $\eta_{\text {stab }}^{*}$ is not only of the size of the critical cluster, it is also the critical cluster, stabilized by the confinement effect.

A Single Cluster Window. How reliable is our "single nucleation event" hypothesis? To test its limits, we reformulate eq 11 for the following case: the synchronized nucleation and growth of two clusters, which is almost a statistically impossible event, and therefore the most unfavorable case for our hypothesis. We can then construct, in the same way as for a single event, the folded critical cluster curve for either of the two events, eq 11 then becoming

$$
\beta_{0}=\beta\left(n^{*}\right)+2 \frac{n^{*}}{N_{\mathrm{s}}}
$$

Equation 10, giving the initial supersaturation $\beta_{\text {crit }}$ above which these two synchronized clusters can nucleate, is given by the following modification

$$
\frac{\partial n^{*}}{\partial \beta_{0}}=-\frac{1}{2} N_{\mathrm{s}}
$$

This minimal $\beta_{\text {crit }}$ is obviously higher than for the single event, and is shown in Figure 3 as the " 2 nucleus limit", lying at $\beta_{0}=$ 2.233. Our hypothesis is thus strictly valid below this supersaturation limit. Its validity can only be extending to higher values on the base of kinetic or statistical considerations which will not be discussed here.

\section{Discussion}

The special case of crystallization of proteins in solution presented here can easily be translate to other kinds of materials (atomic or molecular systems, viruses, colloids). It thus offers a way to fine-tune the size $\left(\eta_{\text {stab }}^{*}\right)$ of the generated crystal, provided the droplet volume can be accurately controlled. Here we take the value $N_{\mathrm{s}}=1 \times 10^{4}$, to reveal the confinement effect: this droplet is subfemtoliter in our solution conditions, femtoliter being equivalent to $N_{\mathrm{s}}=1.2875 \times 10^{5}$. But as we have said, volume will be directly related to solubility of the material, so that the range of volumes where confinement occurs for materials other than the lysozyme presented here cannot be predicted. However, technologies such as microfluidics, ${ }^{24-27}$ microfabricated arrays,${ }^{28}$ emulsions,,${ }^{29,30}$ and nanoscale "dispensers" ${ }^{31,32}$ allow a great range of volumes to be generated. These technologies could offer ways to take advantage of here presented results. First, as already said, to obtain one single crystal per droplet, thus coming from a single nucleation event. Second, and microfluidics particularly fits this goal, nucleation and growth can be totally decoupled: several droplets, where nucleation cannot occur, can be successively added to a droplet containing a single cluster. In so doing, nucleation and growth being totally separated, the properties of the "growing droplets" can be fine-tuned. We thus arrive at a crystal of scalable size, emerging from one single nucleation event. Third, this confinement effect provides a straightforward way to test accuracy of 
Oswald ripening models: by combining two droplets, each containing a crystal of different size, the experiment is made simple.

Results presented here rely on the new and straightforward explanation we offer of the extrema arising from confinement: they are due to the folding of critical cluster size curve. Starting from classical nucleation theory, we found a simpler method (eqs 10 and 11) relying only on the existence of a critical size and the evolution during the nucleation and growth process of the driving force $\Delta \mu$. This does not even depend on the form of the critical cluster size curve, which could differ widely without invalidating our method and results. This led us to affirm their highly model-free aspects. Although results presented here are clearly not more quantitative than could be produced by classical nucleation theory, they offer a clear explanation of the underlying physics, and making it possible to simply evaluate whether confinement should be taken into account. This approach can be used for all first-order phase transitions, provided the " $N_{\mathrm{s}}$ " term can be expressed.

Finally, we identify a clear window of parameters where the nucleation event is alone. This window is defined with the aid of thermodynamics alone. Thus, it gives rise to one single and stable critical cluster. It contrasts with usual experimentations at the scale of critical clusters, which have to monitor the overall evolution of an uncontrolled distribution of precritical, critical and postcritical clusters. Even if impressive results ${ }^{9}$ have been extract from such experiments, confinement effects would be of great help: it provides a new way to study critical clusters and nucleation to test the related models without any interference from kinetics or other clusters. The stability of the generated cluster will, for the first time, allow direct extensive experimentation.

Acknowledgment. We thank Dr. R. Morin, Dr R. Pellenq and Dr. N. Asherie for discussions and comments, without forgetting M. Sweetko for English revision. We thank ANR06-Blan-0355 "MICROCRISTAL" for financial support.

\section{References}

(1) Bentley, W. A.; Humphreys, W. J. Snow Crystals; McGraw-Hill: New York, 1931

(2) Cushing, B. L.; Kolesnichenko, V. L.; O'Connor, C. J. Chem. Rev. 2004, 104, 3893-3946.

(3) Bauer, J.; Spanton, S.; Henry, R.; Quick, J.; Dziki, W.; Porter, W.; Morris, J. Pharm. Res. 2001, 18, 859-866.
(4) Doye, J. P. K.; Poon, W. C. K. Curr. Opin. Colloid Interface Sci. 2006, 11, 40-46.

(5) Seinfeld, J. H.; Pandis, S. N. Atmospheric Chemistry and Physics: From Air Pollution to Climate Change, 2nd ed.; John Wiley \& Sons: Hoboken, NJ, 2006; pp 489-536.

(6) Kashchiev, D. In Nucleation; Butterworth-Heinemann: Oxford, U.K., 2000; pp 17-44.

(7) Baidakov, V. G.; Boltashev, G. S.; Schmelzer, J. W. P. J. Colloid Interface Sci. 2000, 231, 312-321.

(8) Tolman, R. C. J. Chem. Phys. 1949, 17, 333-337.

(9) Gasser, U.; Weeks, E. R.; Schofield, A.; Pusey, P. N.; Weitz, D. A. Science 2001, 292, 258-262.

(10) Auer, S.; Frenkel, D. Nature 2001, 409, 1020-1023.

(11) Lomakin, A.; Asherie, N.; Benedek, G. B. Proc. Natl. Acad. Sci. U.S.A. 2003, 100, 10254-10257.

(12) Moroni, D.; ten Wolde, P. R.; Bolhuis, P. G. Phys. Rev. Lett. 2005, 94, 235703.

(13) Kashchiev, D.; Vekilov, P. G.; Kolomeisky, A. B. J. Chem. Phys. 2005, 122, 244706.

(14) Chernov, A. A. Phys. Rep. 1997, 288, 61-75.

(15) Forsythe, E. L.; Judge, R. A.; Pusey, M. L. J. Chem. Eng. Data 1999, 44, 637-640.

(16) Vekilov, P. G.; Monaco, L. A.; Thomas, B. R.; Stojanoff, V.; Rosenberger, F. Acta Crystallogr., Sect. D 1996, 52, 785-798.

(17) Prinsen, P.; Odijk, T. J. Chem. Phys. 2006, 125, 074903.

(18) Rao, M.; Berne, B. J. Astrophys. Space Sci. 1979, 65, 39-46.

(19) Vogelsberger, W. J. Colloid Interface Sci. 1982, 88, 17-28.

(20) Schmelzer, J.; Ulbricht, H. J. Colloid Interface Sci. 1987, 117, 325338.

(21) Schweitzer, F.; Schimansky-Geier, L. J. Colloid Interface Sci. 1987, 119, 67-73.

(22) Hartman, P. Crystal Growth: An Introduction; North-Holland Publishing Company: Amsterdam, 1973; pp 358-366.

(23) Andreazza, P.; Lefaucheux, F.; Mutaftschiev, B. J. Cryst. Growth 1988, 92, 415-422.

(24) Jahn, A.; Vreeland, W. N.; Gaitan, M.; Locascio, L. E. J. Am. Chem. Soc. 2004, 126, 2674-2675.

(25) Squires, T. M.; Quake, S. R. Rev. Mod. Phys. 2005, 77, 977.

(26) Li, L.; Mustafi, D.; Fu, Q.; Tereshko, V.; Chen, D. L.; Tice, J. D.; Ismagilov, R. F. Proc. Natl. Acad. Sci. U.S.A. 2006, 103, 19243-19248.

(27) Christopher, G. F.; Anna, S. L. J. Phys. D: Appl. Phys. 2007, 40, R319-R336.

(28) Rondelez, Y.; Tresset, G.; Tabata, K. V.; Arata, H.; Fujita, H.; Takeuchi, S.; Noji, H. Nat. Biotechnol. 2005, 23, 361-365.

(29) Utada, A. S.; Lorenceau, E.; Link, D. R.; Kaplan, P. D.; Stone, H. A.; Weitz, D. A. Science 2005, 308, 537-541.

(30) Griffiths, A. D.; Tawfik, D. S. Trends Biotechnol. 2006, 24, 395-402.

(31) Laforge, F. O.; Carpino, J.; Rotenberg, S. A.; Mirkin, M. V. Proc. Natl. Acad. Sci. U.S.A. 2007, 104, 11895-11900.

(32) Fang, A.; Dujardin, E.; Ondarçuhu, T. J. Phys.: Conf. Ser. 2007, 61, 298-301.

CG801165B 